\title{
Immunohistochemical characteristics of the Implant-Hosted Bone: Preliminary findings of 9 mandibular cores
}

\author{
Salah Sakka ${ }^{1}$, Omar Kujan ${ }^{2}$ \\ ${ }^{1}$ DDS, MSc, PhD: Senior Lecturer in Oral and Maxillofacial Surgery, The University of Albaath, School of Dentistry, Hamah, \\ Syria \\ ${ }^{2}$ DDS, MSc, PhD: Senior Lecturer in Oral Pathology, The University of Albaath, School of Dentistry, Hamah, Syria
}

Correspondence:

Abdulhameed Droubi St

Plaza Building, Floor 2

PO Box 2137 Homs, SYRIA

salah.sakka@hotmail.com

\author{
Sakka S, Kujan O. Immunohistochemical characteristics of the Implant- \\ Hosted Bone: Preliminary findings of 9 mandibular cores. Med Oral Patol \\ Oral Cir Bucal. 2011 Mar 1;16 (2):e195-9. \\ http://www.medicinaoral.com/medoralfree01/v16i2/medoralv16i2p195.pdf

Article Number: $16869 \quad$ http://www.medicinaoral.com/
C Medicina Oral S. L. C.I.F. B 96689336-pISSN 1698-4447 - eISSN: 1698-6946
eMail: medicina@medicinaoral.com
Indexed in:
Science Citation Index Expanded
Journal Citation Reports
Index Medicus, MEDLINE, PubMed
Scopus, Embase and Emcare
Indice Médico Español

Received: $25 / 02 / 2010$

Accepted: 25/03/2010

\begin{abstract}
Objectives: The aims of this study were to investigate the biochemical topography of collagen types I and III and to describe the histological structure at the implant placement site to determine the clinical significance of the findings and their possible interaction with bone healing around dental implants.

Material and Methods: Bone cores from 9 mandibles were taken from the site of placement of dental implants. The reliable technique for rapid preparation of fresh-frozen undecalcified bone sections and the indirect immunofluorescent technique as an immunohistochemical procedure were applied. All sections were viewed under U.V light. For comparative purposes the tissue blocks remaining were used for general histology examination.

Results: A homogenous distribution of collagen type I throughout the sections of all cores was evident. Whereas, the distribution of collagen type III throughout the sections of all cores was heterogeneous with different staining patterns. On the other hand, seven cores of the general histology study showed a structure of lamellar mature bone and two cores showed a structure of less mature woven bone.

Conclusion: The biochemical and histological structures of the hosted bone may have an impact on the speed of bone healing around dental implants.
\end{abstract}

Key words: Immunohistochemistry of bone, collagen types I and III, bone healing, dental implants.

\section{Introduction}

'Osseointegration' as a direct anchorage of the implant to the surrounding host bone is the most important aspect to express the reported long-term clinical success of oral implants (1). However, despite high success rate, occurrence of implants failure has been reported (2-5). At this point, the initial biochemical and histological structure of the hosted bone and its possible effect on the healing process around dental implants remains a key question that needs to be answered. Biochemical analysis of bone homogenates and extracts showed that the organic matrix of bonny tissue consist primarily of collagen type I (6) Collagen type III is mentioned only in brief and there is little comment in the literature with 
regard to hard tissues, where it appears transitionally in developing fracture callus (7) and more constantly in regions of woven bone in the mandible (8).

The aim of this study was to investigate the histological structure and biochemical topography of the implanthosted bone with special regard to collagen types I and III. The latter plays a significant role during the process of bone healing and intra membranous ossification $(9,10)$.

\section{Materials and Methods}

With patients consent, bone cores from 9 mandibles (Table 1) were taken from the site of placement of oral implants using IMZ-trephine ( $3.1 \mathrm{~mm})$.

Sections $10 \mu \mathrm{m}$ thick of fresh frozen bone were prepared according to the method for undecalcified bone of Aaron and Carter (1987) (11) using an LKB PMV 2258 heavy duty cryomicrotome (Palmstiernas Instrument; Stockholm, Sweden).

Indirect immunofluorescent technique was performed as an immunohistochemical Study. All sections were incubated in primary antibody, which had been diluted in phosphate-buffered saline (PBS, PH 7.2) for 30-40 minutes. Primary monoclonal antibodies, which had been raised in mouse against human collagen type I, 1:50; (ICN Biomedicals; high wycombe, UK) and against human collagen type III, 1:50; (BioGenex, San Ramon, USA) were used. Fluorescein-conjugated antibody in the form of fluorescein isothiocyanate FITC-conjugated rabbit anti mouse (Dako, DENMARK) diluted 1:40 for 30 minutes was used as the secondary antibody for localizing collagen types I and III. Finally all sections were washed in PBS, PH 7.2 and mounted in immumount and then viewed under U.V light. Double blinded assessment of the immunostaining patterns of the examined slides was done by two observers (S.S, O.K) and consensus scores reached in discrepant cases.

The tissues remaining were fixed in cold puffered formalin, $\mathrm{pH} 7.0$, for 3-6 days then demineralised in $20 \%$ tetrasodium ethylenediaminetetraacetic acid (EDTA, $\mathrm{pH}$ 7.0), before wax embedding, sectioning, and staining with hematoxilin-eosin H\&E, for comparative purposes.

Table 1. Patients descriptive data.

\begin{tabular}{|c|c|c|c|c|}
\hline Patient number & Sex & Age & Mandible & Site of biopsy \\
\hline 1 & F & 73 & Severly Resorbed & 43 \\
\hline 2 & M & 55 & Severly Resorbed & 31 \\
\hline 3 & F & 24 & Adequate Bone Level & 35 \\
\hline 4 & F & 59 & Severly Resorbed & 41 \\
\hline 5 & F & 51 & Severly Resorbed & 31 \\
\hline 6 & M & 68 & Adequate Bone Level & 44 \\
\hline 7 & M & 63 & Adequate Bone Level & 32 \\
\hline 8 & F & 60 & Adequate Bone Level & 32 \\
\hline 9 & F & 56 & Severly Resorbed & 41 \\
\hline
\end{tabular}

\section{Results}

Collagen type I distribution:

The distribution of collagen type I throughout the sections of all cores was homogenous and strong staining was evident (Fig. 1), (Table 2).

Collagen type III distribution:

The distribution of collagen type III throughout the sections of all cores was heterogeneous and different staining patterns were observed (Fig. 2 A-C); (Table 2).

General histology

Different bone structures were found. Seven cores showed a structure of mature lamellar bone type and two cores showed less mature woven bone type.

(Fig. 3A) illustrates the structure of bone in polarized light and shows concentric lamellae confirming the mature nature of the bone matrix. The surface shows little activity. Within the marrow, small peripheral nerve bundle seen. Fibrous band is present indicating healing repair process has taken place.

(Fig. 3B) illustrates the structure of bone in polarized light where most of the trabeculae show a woven bone matrix. The bone is less mature and the trabeculae lack orientation and connectivity. The section also shows small piece of old bone upon which new bone has been laid down. Prominent reversal line is present at the junction between the old and new bone. There is evidence of surface resorption and formation.

Table 2. The distribution of collagen type I and III.

\begin{tabular}{|c|c|c|}
\hline Core no. & Collagen type I & Collagen type III \\
\hline 1 & +++ & - \\
\hline 2 & +++ & ++ \\
\hline 3 & +++ & + \\
\hline 4 & +++ & + \\
\hline 5 & +++ & + \\
\hline 6 & +++ & + \\
\hline 7 & +++ & + \\
\hline 8 & +++ & - \\
\hline 9 & +++ & \\
\hline
\end{tabular}

(-): Absent

$(+)$ : Evident in less $50 \%$ of the field

$(++)$ : Evident in over $50 \%$ of the field

$(+++)$ : Evident in $100 \%$ of the field 


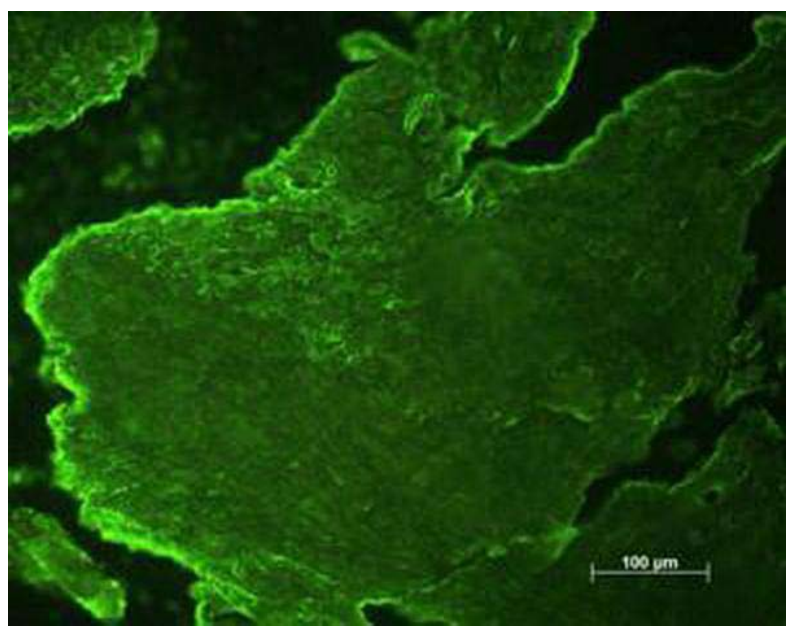

Fig. 1. Photomicrograph of a section from core one showing general staining of the bone matrix, where type I collagen was evident in $100 \%$ of the field.

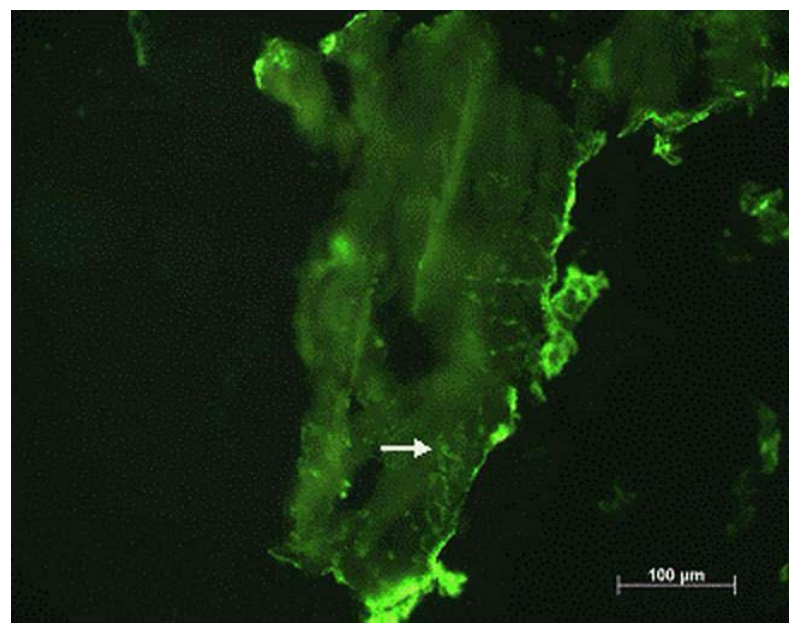

Fig. 2B. Photomicrograph of a section from core six, where collagen III (arrow) was evident in less than $50 \%$ of the field.

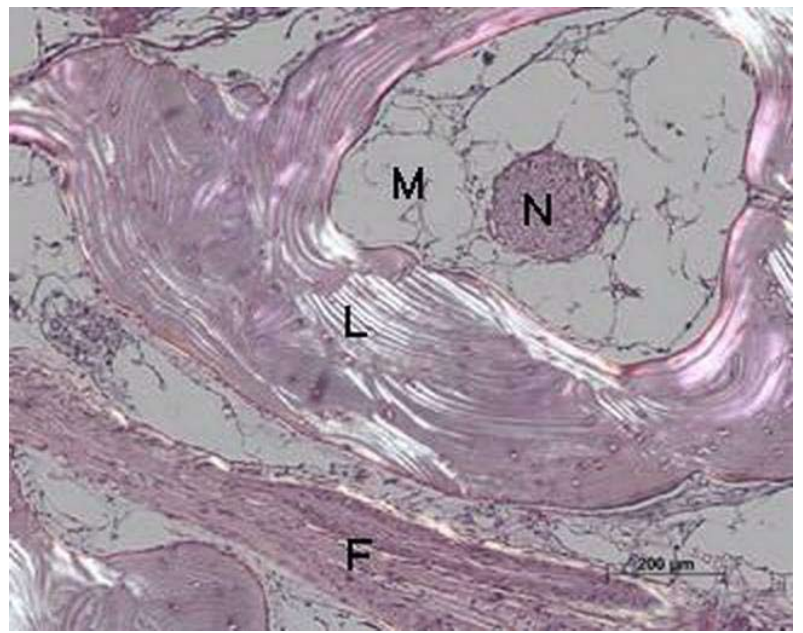

Fig. 3A. Photomicrograph of a section from core one in polarized light showing the mature trabeculae. $\mathrm{F}=$ Fibrous Band, $\mathrm{L}=$ Mature Lamellae, $\mathrm{M}=$ Bone Marrow, $\mathrm{N}=$ Nerve Bundle.

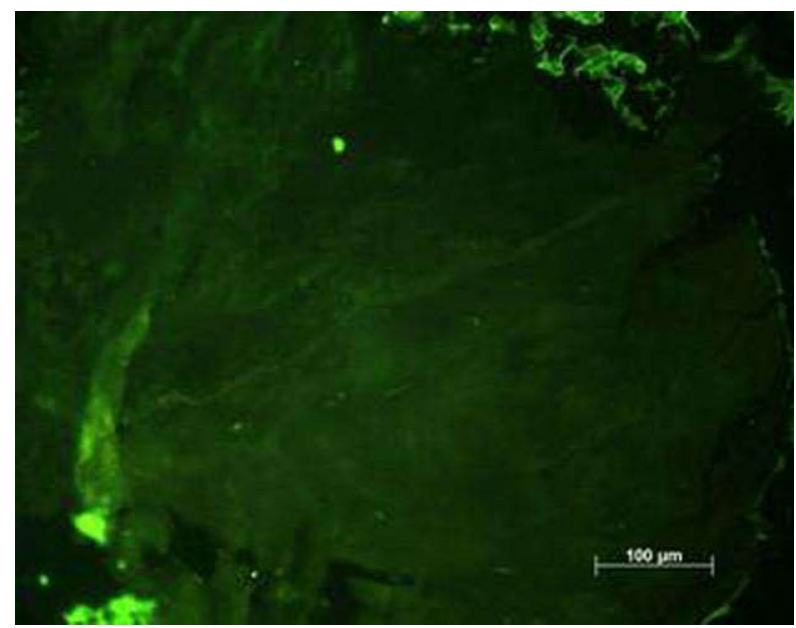

Fig. 2A. Photomicrograph of a section from core nine showing the absence of type III collagen.

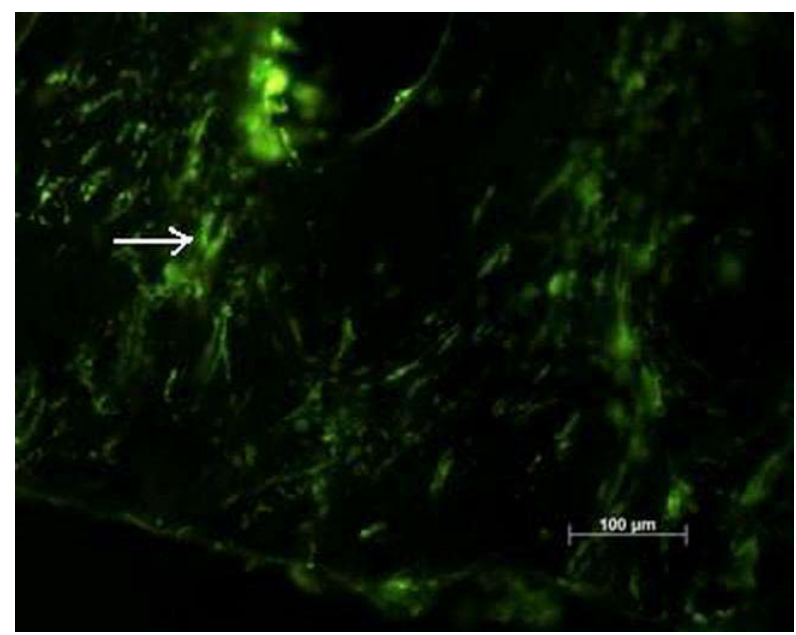

Fig. 2C. Photomicrograph of a section from core three, where collagen III (arrow) was evident in over $50 \%$ of the field.

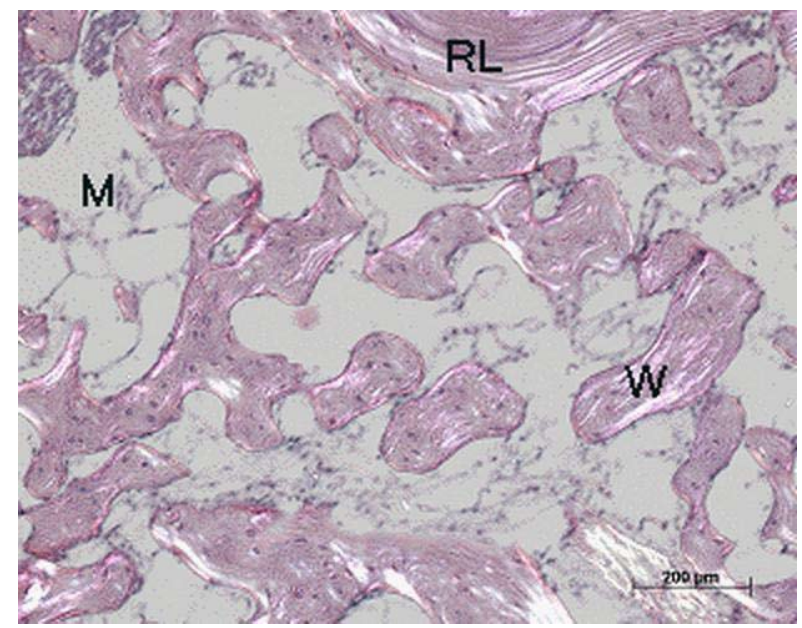

Fig. 3B. Photomicrograph of a section from core three in polarized light showing the irregular trabeculae pattern. $\mathrm{RL}=$ Reversal line, $\mathrm{M}=$ Bone Marrow, $\mathrm{W}=$ the immature (woven) bone. 


\section{Discussion}

The initial healing period of bone around oral implants seems to be of great importance to achieve the direct anchorage of implants into the surrounding host bone, which is the primary goal for implants success. This study was carried out in an attempt to investigate the relationship between the histochemical structures of the jaw-bones and their subsequent healing around oral implants .

Antibodies to collagen fibres have proved to be extremely valuable in determining the distribution of different collagen types in various connective tissues. Specific antibodies to human types I and III collagens were used in fresh frozen sections of human alveolar and basal bone prepared using the technique described by (Aaron and Carter, 1987) (11). This technique is a reliable technology for preparation of thin fresh frozen bone sections for optical microscopy under UV illumination. Such technique preserves the biochemistry of the tissue and enables the application of immunohistochemical methods to the extracellular components of the bone matrix.

Among the collagens, type I has been most intensively studied and has been shown to be an omnipresent constituent of dentine, periodontium and alveolar bone $(8$, 12-14). The results presented in this study showed that type I collagen was homogeneously distributed in all sections where fibres were arranged in regular organization (Fig. 1).

On the other hand, studies show that collagen type III fibres bear a striking similarity to Sharpey's fibres with regards to their morphology, their immunohistochemical staining patterns (15), and their arrangement at the cement line (16). The role of Collagen type III fibres during the process of bone healing and intramembranous ossification is important $(9,10)$. These fibres are able to form quick crosslinks and to stabilise the repair site (9). It has also been reported that the peripheral trabeculae of the human femoral anlagen are formed of coarse fibres, which are recognised by antibodies raised against collagen type III (17). The arrangement of these fibres suggests that they form a preliminary framework on which the process of intramembranous ossification proceeds. The results presented in our study showed that there was heterogeneity in the distribution of collagen type III throughout the sections (Figs. 2A-C) indicating that the alveolar bone may contain more Sharpey's like-fibres whereas basal bone contained few or no such fibres.

General histology of the remaining tissues of this study have demonstrated different bone structures. This difference, in respect to bone maturity, seems to be important during the process of bone healing around implants. The mature bone type (Fig. 3A) showed regular trabecular bone pattern with little surface activity.
On the other hand, the immature bone type (Fig. 3B) showed irregular trabecular bone pattern with evidence of surface resorption and formation, which may indicate that the tooth socket may still be undergoing healing process.

The immature bone is more dynamic where active remodelling process leads to rapid bone formation (18). Whereas, mature bone is slowly formed and this formation will be slower with increasing maturation (19).

In Summary, The difference in bone biochemical topography, in terms of the heterogeneity of the distribution of collagen type III, and histological structure, in terms of maturity, may have an impact on the speed of bone healing around dental implants. The small number of the bone cores limited the study. Therefore, no statistical analysis was done. However, the results were exciting and further research will be indicated for definite answer.

\section{References}

References with links to Crossref - DOI

1. Brånemark PI, Hansson BO, Adell R, Breine U, Lindström J, Hallén $\mathrm{O}$, et al. Osseointegrated implants in the treatment of the edentulous jaw. Experience from a 10-year period. Scand J Plast Reconstr Surg Suppl. 1977;16:1-132.

2. Lekholm U, Van Steenberghe D, Herrmann I, Bolender C, Folmer $\mathrm{T}$, Gunne J, et al. Osseointegrated implants in the tratment of partially edentulous jaws: A prospective 5-year multicentre study. Int $\mathrm{J}$ Oral Maxillofac Implants. 1994;9:627-635.

3. Friberg B, Nilson H, Olsson M, Palmquist C. Mk II: the selftapping Brånemark implant: 5-year results of a prospective 3-center study. Clin Oral Implants Res. 1997;8:279-85.

4. Jemt T, Häger P. Early complete failures of fixed implant-supported prostheses in the edentulous maxilla: a 3-year analysis of 17 consecutive cluster failure patients. Clin Implant Dent Relat Res. 2006;8:77-86.

5. Schwartz-Arad D, Laviv A, Levin L. Failure causes, timing, and cluster behavior: an 8-year study of dental implants. Implant Dent. 2008;17:200-7.

6. Veis A. Bones and teeth. From Extracellular matrix biochemistry. New York: Elsiver; 1984 .p. 329-74

7. Page M, Hogg J, Ashhurst DE. The effects of mechanical stability on the macromolecules of the connective tissue matrices produced during fracture healing. I. The collagens. Histochem J. 1986;18:25165 .

8. Becker J, Schuppan D, Benzian H, Bals T, Hahn EG, Cantaluppi $\mathrm{C}$, et al. Immunohistochemical distribution of collagens types IV, V, and VI and of pro-collagens types I and III in human alveolar bone and dentine. J Histochem Cytochem. 1986;34:1417-29.

9. Liu SH, Yang RS, al-Shaikh R, Lane JM. Collagen in tendon, ligament, and bone healing. A current review. Clin Orthop Relat Res. 1995;318:265-78

10. Carter DH, Sloan P, Aaron JE. Immunolocalization of collagen types I and III, tenascin, and fibronectin in intramembranous bone. J Histochem Cytochem. 1991;39:599-606.

11. Aaron JE, Carter DH. Rapid preparation of fresh-frozen undecalcified bone for histological and histochemical analysis. J Histochem Cytochem. 1987;35:361-9.

12. Tung PS, Domenicucci C, Wasi S, Sodek J. Specific immunohistochemical localization of osteonectin and collagen types I and III in fetal and adult porcine dental tissues. J Histochem Cytochem. 1985;33:531-40.

13. Becker J, Schuppan D, Rabanus JP, Rauch R, Niechoy U, Gelderblom HR. Immunoelectron microscopic localization of collagens 
type I, V, VI and of procollagen type III in human periodontal ligament and cementum. J Histochem Cytochem. 1991;39:103-10.

14. Huang YH, Ohsaki Y, Kurisu K. Distribution of type I and type III collagen in the developing periodontal ligament of mice. Matrix. 1991;11:25-35.

15. Wang HM, Nanda V, Rao LG, Melcher AH, Heersche JN, Sodek J. Specific immunohistochemical localization of type III collagen in porcine periodontal tissues using the peroxidase-antiperoxidase method. J Histochem Cytochem. 1980;28:1215-23.

16. Johnson RB. A classification of Sharpey's fibers within the alveolar bone of the mouse: a high-voltage electron microscope study. Anat Rec. 1987;217:339-47.

17. Carter DH, Sloan P, Aaron JE. Trabecular generation de novo. A morphological and immunohistochemical study of primary ossification in the human femoral anlagen. Anat Embryol (Berl). 1992;186:229-39.

18. Weinlaender M. Bone growth around dental implants. Dent Clin North Am. 1991;35:585-601.

19. Roberts WE. Bone tissue interface. J Dent Educ. 1988;52:804-9. 